\title{
Some Statistical Properties of Pulsed Oblique HF Ionospheric Transmissions
}

\author{
Martin Balser and William B. Smith
}

\author{
Contribution From Lincoln Laboratory, * Massachusetts Institute of Technology, Lexington 73, Mass.
}

(Received April 6, 1962; revised May 7, 1962)

\begin{abstract}
A study is made of the amplitude fading of 35- $\mu$ sec HF pulses transmitted via the ionosphere over a 1,566-km path between Atlanta, Ga., and Ipswich, Mass. The distribution functions for about half of the records taken fit the family of distributions for a sine wave in Gaussian noise, with most of these best fitting the curve for pure noise (the Rayleigh distribution). Some examples of two sine waves with random phase are observed. It is concluded that most of the remaining curves which do not fit these distributions correspond to samples of nonstationary functions. The median fading time for one-hop paths is of the order of 20 seconds, and many examples occur with considerably longer fading times. Multiple-hop paths give fading times of a very few seconds. Space correlation distances are also considerably greater than expected, averaging around 40 wavelengths, which corresponds to a mean angular deviation of the order of two tenths of a degree. Many crosscorrelation functions show peaks displaced in time from the origin, reflecting the effect of ionospheric winds.
\end{abstract}

\section{Introduction}

Many papers, too numerous to cite individually, have been written on some theoretical or experimental aspect of the statistics of fading $\mathrm{HF}$ ionospheric signals. (A goodly number of these are listed in the Bibliography on Ionospheric Propagation of Radio Waves [Nupen, 1960] under Statistical Analysis and Fading Pattern.) Still, many questions remain on relatively elementary statistical characteristics such as the first order distribution of the amplitude (Rayleigh or not?) and correlation functions in space and time.

Most of the research work in the field, particularly that on ionospheric roughness and winds, appears to have been done at vertical incidence. Much of the oblique transmission, which more nearly approximates conditions on communication circuits, used CW signals, which obscured the individual characters of and differences between the different propagation paths. Furthermore, results rarely could be related to the propagation paths, since these were generally not identified.

Accordingly, a program of measurement of the amplitude statistics of HF oblique transmissions was set up at Lincoln Laboratory to try to fill in some of these gaps and, if possible, to relate the results to propagation theories. Terminals were established at Ipswich, Mass., and Atlanta, Ga., a distance apart of $1,566 \mathrm{~km}$. This distance, while it is not as long as most communication circuits of interest, allows the observation of one-hop $E$ and $F$ propagation as well as multiple-hop. Transmissions were pulsed to separate the signals arriving via different ionospheric layers or heights, and the fixed-frequency transmissions used for taking data were

\footnotetext{
*Operated with support from the Army, Navy, and Air Force.
}

interspersed with sweep-frequency ionograms which were used to identity the paths observed at fixed frequency. Finally, a fairly involved data processing unit was designed and built so that rather large quantities of data could be recorded digitally at the field site and be ready for digital computer processing to produce the desired information.

\section{Equipment and Experimental Procedure}

The radio equipment, identical at both ends, consisted essentially of synchronized stepped-frequency Phillips sounders, which covered the range 1 to $25 \mathrm{Mc} / \mathrm{s}$ in $100 \mathrm{kc} / \mathrm{s}$ steps, plus some control equipment. All timing and radiotrequencies were derived from Loran standards so that synchronization, once established, would hold for many hours. Pulses of Gaussian shape, 35- $\mu$ sec duration and about $10-\mathrm{kw}$ peak power were transmitted 15 times per second. Each terminal was equipped with a rhombic antenna $300 \mathrm{ft}$ on a leg and $70 \mathrm{ft}$ high. These characteristics produced a peak gain of 16 to $22 \mathrm{db}$ over the frequency range 8 to $20 \mathrm{Mc} / \mathrm{s}$. Even at the lower end of this range, the gain at the nominal E-layer takeoff angle of $4^{\circ}$ was only a little over $10 \mathrm{db}$ down from the peak, which was chosen to coincide with normal $F$-layer takeoff angles in the center of the frequency range. The computed antenna pattern was verified by direct measurement at a representative frequency.

For these statistical observations, two modes of operation were employed. In both cases, pulses were transmitted at Atlanta and the data recording was done at the receiving site in Ipswich. In the first mode, the signal was received on the rhombic antenna. This gave the advantage of the large gain which made easier the task of obtaining usable data (a signal-tointerference ratio of $20 \mathrm{db}$ was set as the minimum 
required to produce clean statistical results). Also, using the data processing equipment described below, several paths appearing in the received signal could be examined simultaneously, thus making information available on many different propagation paths in a relatively short time interval. In the other mode, the correlation of amplitude fading with distance was studied. For this purpose, six vertical whip antennas $23 \mathrm{ft}$ high were erected at varying separations along a line perpendicular to the propagation path.

All of the data recording referred to above was done on fixed frequency transmissions. Interspersed among the periods of data taking, sweepfrequency ionograms were recorded. The principal function of these ionograms was as an aid in identifying the paths being studied. It is quite difficult ordinarily, given a received signal at a given frequency comprising perhaps three or four pulses, to determine the propagation path by which the energy traveled. This task can be considerably simplified by examining the overall pattern of the traces in an ionogram. Figure 1 shows two ionograms that are representative of almost all of the days on which the system operated. Typical of winter days is figure 1a, taken at 1239 EST, 28 December 1959. The interpretation drawn just below the original ionogram indicates the paths corresponding to each trace. (Dotted lines are drawn in to complete the pattern in two of the traces. These dropouts occur at frequencies for which the rhombic antenna pattern has a nuil at the takeoff angle required for the indicated path, thereby lending support to the path identification given.) Thus, for instance, the received signal at about 17 $\mathrm{Mc} / \mathrm{s}$, consisting of two pulses about $200 \mu \mathrm{sec}$ apart followed a millisecond later by a third pulse can be seen to be the $E, F$, and two-hop $F$ paths. The two pulses which would appear at $23 \mathrm{Mc} / \mathrm{s}$ are here seen to be the low and high one-hop $F$ paths. (Although it does not show on this ionogram, the high ray path was commonly resolved into the two magneto-ionic components.) Figure 1b, taken at 1433 on 18 May 1960, typifies summer conditions. The notable differences are the higher MUF of the $E$ relative to the $F$ return and the splitting of the $F$ into $F_{1}$ and $F_{2}$ returns.

The records used in this study were of duration 5 to $20 \mathrm{~min}$, with most being 10 or $15 \mathrm{~min}$ long. Usable records of about 150 different paths were obtained on days scattered throughout the period October 1959 to July 1960.

\section{Data Processing}

The heart of the data processing scheme for this program was a unit which was especially designed and constructed to record the data at the field site onto magnetic tape in digital format compatible with input requirements of the IBM 709 computer at the laboratory. Six channels were measured and recorded independently. The process was initiated by a pulse synchronized with the transmitter pulse. Each channel was supplied with a $10-\mu$ sec sample pulse placed at will at any time within 10 msec of the original pulse, this time being independently determined for each channel. The maximum voltage of the signal in the channel during the sample pulse was then stored for the remainder of the $10-\mathrm{msec}$ period. At the end of that time, the six stored voltages were rapidly converted in sequence to digital form and the six most significant bits in each number were stored in a digital storage, thus defining each voltage as one of 64 possible levels. These stored numbers were read out in sequence, one every $1 / 90$ sec, onto a continuously moving tape. Thus one machine word, 6 rows of 6 bits each, contained all the information given in the six channels corresponding to one received pulse, and this procedure was repeated for each pulse.

In particular, for the first mode of operation described in the preceding section, the inputs to all six channels were connected to the output of the single receiver being fed by the rhombic antenna. The sample pulses in the individual channels were placed at the various peaks in the received pulse train. Thus all paths contributing energy at the frequency being employed were studied simultaneously. For the other, or space correlation, mode of operation, the six antennas each fed a separate receiver. These receivers served as the inputs to the six channels, and a single path was studied at any given time by sampling all the channels simultaneously, corresponding to the time of arrival of the given path. (All the receivers, incidentally, had linear outputs to simplify the processing problem.)

A program was written to process this raw data on an IBM 709 digital computer. Basically, the program obtained for every record the first order distribution and the autocorrelation function for each channel individually, and the crosscorrelation functions for all pairs of channels. Because of the enormous amount of machine time which would have been required for the full operation indicated, options were included to use only the parts of the program appropriate to each record or to edit the original tape down to a grid of points separated by times comparable to the correlation time. Despite these savings, a considerable quantity of time, amounting to the equivalent of almost $40 \mathrm{hr}$ on the 709 computer, was required to obtain the indicated information trom the data.

\section{Results-Distributions and Autocorrelations}

The cause of fluctuations in the received field strength of a radio wave is generally assumed to be the changing configuration of inhomogeneities in the intervening medium, in this case the ionosphere. The wave equation

$$
\nabla \times \nabla \times E-k^{2} n^{2}(r, t) E=0
$$

can be rewritten

$$
\nabla \times \nabla \times E-k^{2} n^{2}(r) E=k^{2} \Delta n^{2}(r, t) E
$$



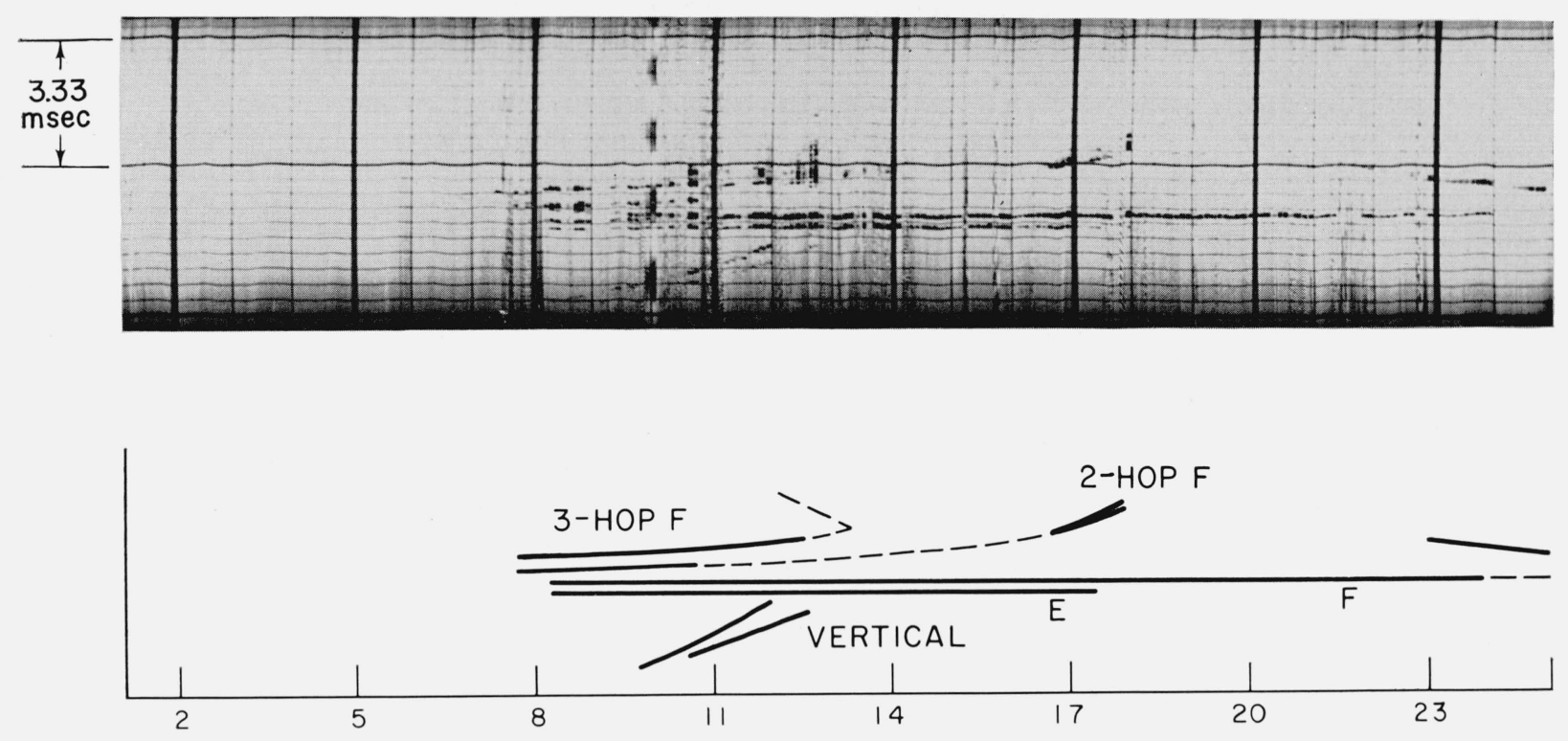

(a)
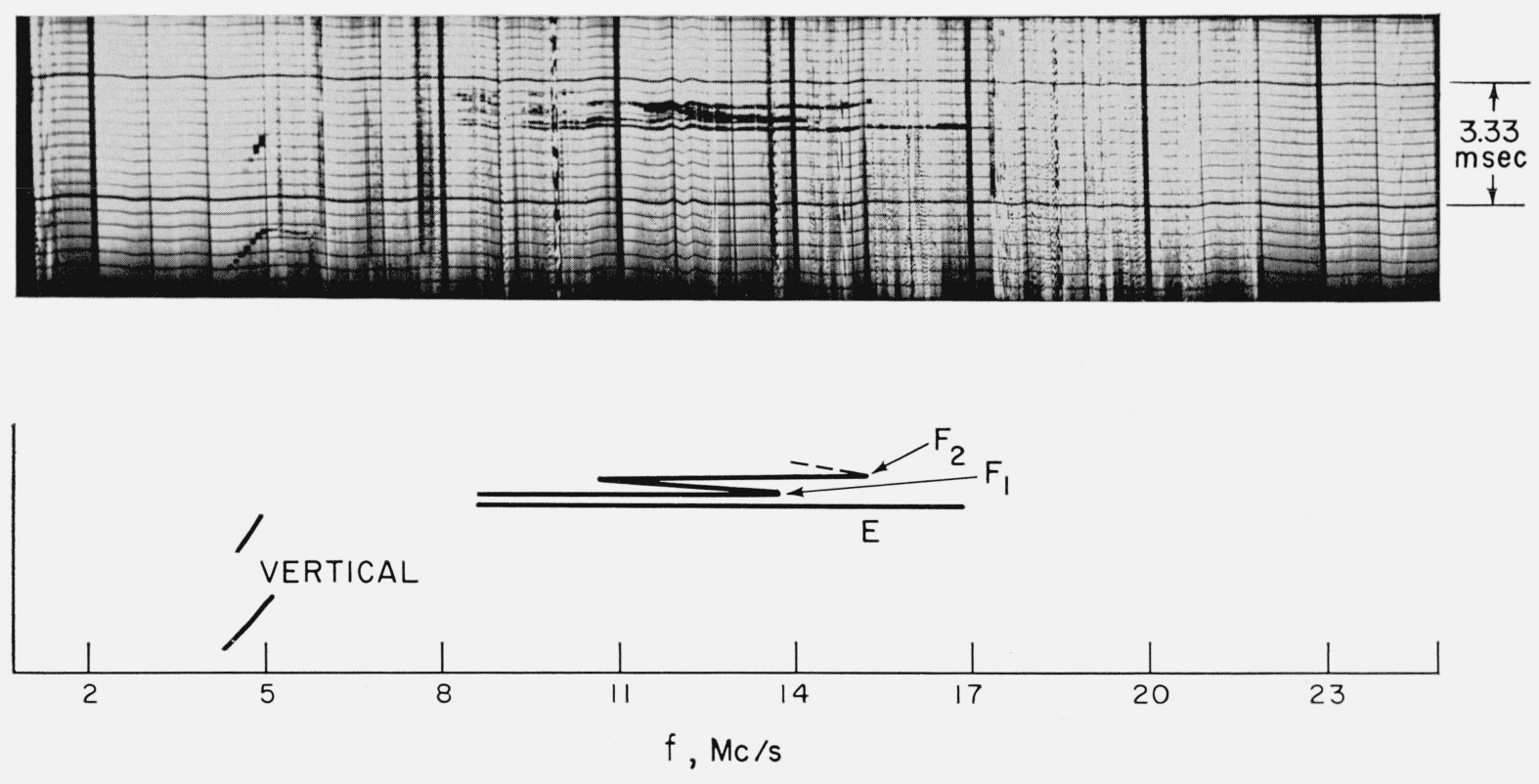

(b)

Figure 1. Typical ionograms, with traced interpretation of path structure.

(a) Winter day (b) Summer day 
to separate the effects of the mean refractive index profile $n(r)$ from the fluctuations $\Delta n$ which are presumed small compared to $n$. The solution of this equation can be formally written as

$$
E^{\prime}(r)=E_{\text {inc }}(r)+\int \Gamma\left(r, r^{\prime}\right) \cdot k^{2} \Delta n^{2}\left(r^{\prime}\right) E\left(r^{\prime}\right) d r^{\prime}
$$

where $\Gamma$ is a Green's function (which would be very complicated indeed for the case of propagation via the ionosphere). For very weak scattering, the field $E$ inside the integral is replaced by the incident field $E_{\text {inc. }}$ The scattered field, represented by the integral, is seen to be a sum over the contributions of all regions of inhomogeneity, presumably covering very many correlation volumes. Thus, by the central limit theorem, this scattered field would be normally distributed. As the amount of scattering increases, the strength of the incident wave (the specular component) would decrease accordingly. (When this has happened to a significant degree, the single scattering approximation fails, but the central-limit argument should apply equally well to the multiple scattering terms which must be added.) Finally, the incident wave would vanish and its energy be transferred completely to the scattered field.

The statistical implication of this argument is that the received field should consist of a sine wave in Gaussian noise. (Here and henceforth, "noise" shall be understood to refer to scattered energy, not to unwanted interference.) The amplitude distribution of such a signal is a member of a one-parameter family of distributions [Rice, 1945], several of which are shown in figure 2. The parameter, $a$, which characterizes a given curve is the ratio of the sine wave amplitude to the rms noise (the signal-to-noise power ratio should therefore be $\left.a^{2} / 2\right)$. Thus the curve for $a=0$ represents pure (scattered) noise, and is the well-known Rayleigh distribution. A pure sine wave $(a=\infty)$ would be represented by a step function. These curves, and all further experimental and theoretical distributions, have for ease of comparison been normalized by redefining the abscissa

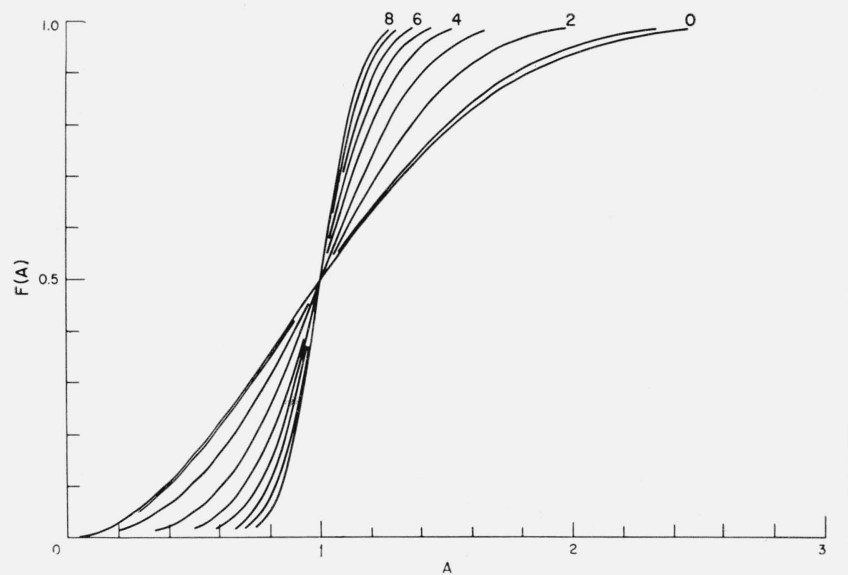

Figure 2. Distributions of the amplitude of a sine wave in Gaussian noise.

Labels are the ratio of sine wave amplitude to rms noise. to be unity where the distribution function is 0.5 .

After those records were eliminated which seemed not to contain valid data (because of excessive interference, path fading out, equipment trouble, etc.), 146 records averaging a little over $10 \mathrm{~min}$ in duration, remained to be processed. First order distributions and autocorrelation functions were computed for each record, and these were plotted by machine, the distributions in the standard format described in the preceding paragraph.

The suspicions, brought about by visual observation of the signal at the field site, that there would difficulty in obtaining clean statistical data, were confirmed by the first results. On the one hand, fading times were much longer than anticipated, and early 5 -min records contained as few as 8 or 10 correlation times. As a result of being derived from so few independent samples, the distribution and correlation curves had much structure which represented statistical fluctuations rather than the desired results. On the other hand, the question of how long the signal could be described as stationary limited the obvious course of taking longer and longer samples. A sample length of about $10 \mathrm{~min}$ was used most commonly, and as will be seen, appears to be a fair compromise.

With some allowance made for these random bumps in the distribution curves, just about half (70) could be said to fit the family of curves in figure 2. (A rather similar proportion was found in a study by Brennan and Phillips [1957] at medium frequency.) Of these, only 20 showed distinct indications of having a specular component $(a \geq 2)$ while the remainder best fit the Rayleigh distribution (or the curve for $a=1$ which is barely distinguishable from it). An example of an experimental Rayleigh distribution (2-11-60, $1232 \mathrm{EST}, 11.35 \mathrm{Mc} / \mathrm{s}$, 2-hop $F)$ is shown in figure 3a. In general, the highest signal-to-noise ratios $(a \geq 3)$ occurred only for frequencies very close to the MUF, scmewhat more for $F$-layer propagation than $E$, somewhat more in the winter than in the summer. The most extreme cases, such as the one illustrated in figure $3 \mathrm{~b}$ (10-12-59, $1556,23.25 \mathrm{Mc} / \mathrm{s}, F$ high ray) with the curve for $a=7$, occurred for high rays in which the magnetoionic modes were resolved. As is clear from the distribution, practically no fading at ali took place over the course of this 10 -min record. All of the multiple-hop records resulted in noise-like (Rayleigh) distributions as did the remainder of the one-hop records.

One small group, consisting of 9 records, deserves ome attention before the bulk of the remaining records is taken up. These curves, an example of which $(10-12-59,1508,13.35 \mathrm{Mc} / \mathrm{s}, F)$ is shown in figure 3c, have a distinctly different shape from the other families considered, and resemble the solid curve in the figure, which is the distribution of the amplitude of two sine waves of equal amplitude with all possible phase shifts equiprobable. Thus, for instance, two unresolved magneto-ionic modes beating against each other in random phase would produce this distribution. The computed curve 


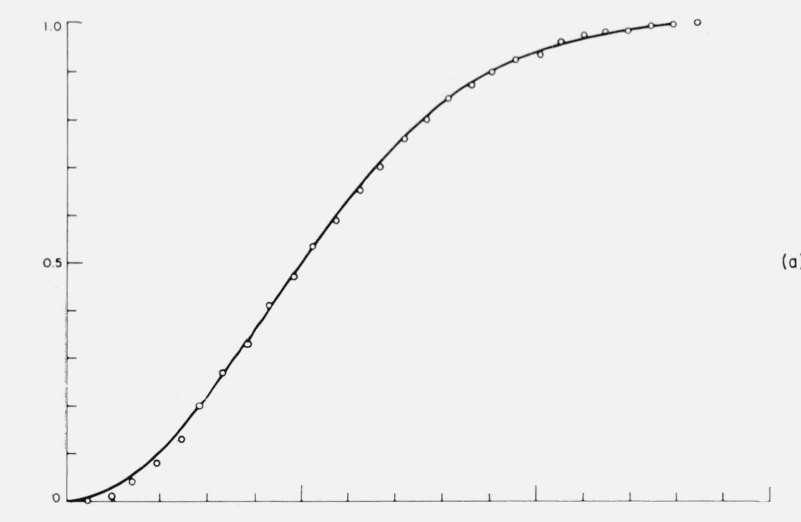

(a)

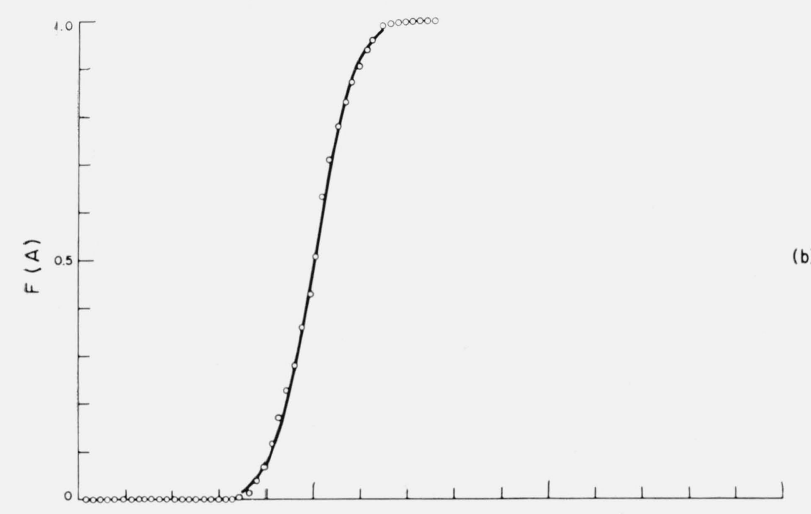

(b)
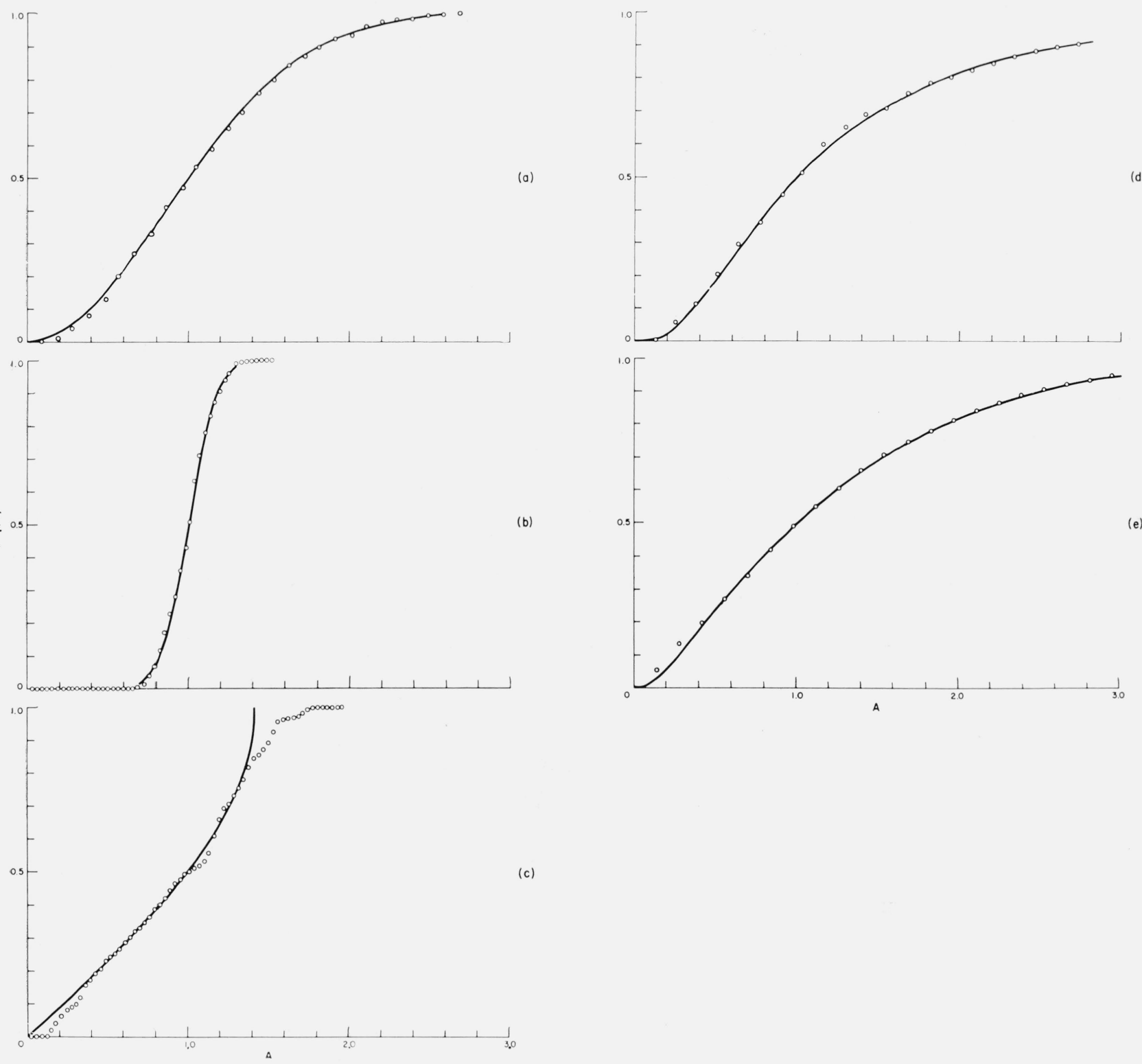

Figure 3. Five experimental distributions.

The solid theoretical curves are (a) $a=0$ (Rayleigh), (b) $a=7$, (c) 2 sine waves, (d) $\sigma=1.1 / \sqrt{2}$, (e) $b=10$. (See text for details.)

assumes no noise, and the experimental curve clearly has some at both ends. Curves for two sine waves of different amplitude and varying amounts of noise would form a two-parameter family (one of which is the set in figure 2) which were not computed. It is therefore possible that some, but probably not many, of the other distributions could have been identified with this family.

These identifications leave nearly half of the total number of records still not associated with a theoretical distribution. A large number of these are clearly distinguished from the set of curves already identified in that they occupy those "quadrants" of the distribution plane (refer to figure 2) on the opposite side of the Rayleigh curve $(a=0)$ from the distributions for $a>0$. One further possibility not yet considered which also has some theoretical justification ['Tatarski, 1961] is the family of log normal distributions. This is basically a two-parameter family of distributions of the quantity $A=A_{0} e x$, where $A_{0}$ is a fixed level and $x\left(=\ln A / A_{0}\right)$ is normally distributed with mean zero and standard deviation $\sigma$. For the present purposes, the family may be considered to depend only on the one parameter $\sigma$, since the "level" parameter $A_{0}$ is automatically compensated for by the standard normalization procedure for distribution functions. This amplitude distribution would be produced if the fading, rather than being caused by scattering, resulted from varying absorption. If $x$ is replaced by 
$-\int \kappa d s$ where $\kappa$ is the absorption coefficient, and $\kappa$ (or simply the integral as a whole) varies normally, then $A$ is seen to vary $\log$ normally. Several curves were drawn corresponding to various values of $\sigma$, and these were compared with the 67 remaining distributions. Indeed, a number of the curves seemed to agree fairly well and some others rather less well. An example wherein $\sigma=1.1 / \sqrt{2}$ is given in figure $3 \mathrm{~d}\left(7-1-60,1032,13.95 \mathrm{Mc} / \mathrm{s}, F_{1}\right)$.

Nevertheless, other considerations tend to indicate that these agreements may well be fortuitous and the interpretation erroneous. First of all, it is doubtful that fairly rapid fading of $10 \mathrm{db}$ and more at these frequencies can regularly be ascribed to absorption. Further, some simultaneous observations made on the rhombic antenna and a vertical whip indicated by their low, or sometimes distinctly negative, correlation that the fading was generally polarization fading, associated with phase changes, rather than absorption fading. Finally, on numerous occasions signals were observed to weaken or strengthen systematically with time. This would suggest that the signal may often be nonstationary and hence not properly described by any of the statistical arguments covered thus far.

In order to test the plausibility of this hypothesis, an example of the distributions which would result from a simple but reasonable nonstationary process was computed. The envelope of a Gaussian process of mean zero and standard deviation $\sigma$ has the Rayleigh frequency function

$$
f_{\sigma}(A)=\frac{A}{\sigma^{2}} e^{-\frac{A^{2}}{2 \sigma^{2}}}
$$

the distribution function (integral) of which has already been discussed. Suppose now that $\sigma$ is increased systematically and linearly in time from $\sigma_{1}$ to $\sigma_{2}$ (for instance by changing a gain control). The measured distribution function for this nonstationary process would be

$$
F(A)=\frac{1}{\sigma_{2}-\sigma_{1}} \int_{0}^{A} \int_{\sigma_{1}}^{\sigma_{2}} f_{\sigma}(A) d \sigma d A
$$

After the standard normalization, this would be a new one-parameter family of distributions, depending only on $b=\sigma_{2} / \sigma_{1}$, in which $b=1$ corresponds to the Rayleigh distribution. This family was also plotted and was found to exhibit the desired property of being the result (crudely speaking) of a clockwise rotation of the Rayleigh distribution about the point of normalization. Many of the experimental distributions were found to agree with this family. An (extreme) example, for $b=10$ may be found in figure 3e $(12-28-59,1107,8.95 \mathrm{Mc} / \mathrm{S}, 2$-hop $F)$. Different modes of variation of $\sigma$ would, of course, result in different distributions having the same general configuration as the bulk of the unidentified curves, including the ones which were found to agree with the log normal curves. It is therefore concluded that 52 distributions can be fairly well fitted with the family just discussed or, having generally similar shapes, can be ascribed to nonstationary functions of this type.

The remaining 15 distributions, which had a very irregular shape or too few points because of very low amplitude, could not be identified at all. These observations are summarized in table 1.

TABLE 1. Summary of observed distribution functions

Sine wave in Gaussian noise:

Rayleigh (pure noise) 50

Specular component $(a \geq 2) \ldots$

Two sine waves $\ldots \ldots$

Probably nonstationary

Unidentifiable $\ldots \ldots$

As previously noted, the normalized autocorrelation function was also computed for each record. Examination of these curves bore out the field observation that the correlation time was in many cases much longer than anticipated. This condition has the unfortunate effect, already discussed in relation to the distribution function, of making it difficult to estimate accurately a statistical characteristic during a period for which the process can be considered stationary. The same consideration applies to the correlation function itself, so that it is difficult even to determine the correlation time when it is long.

Some feeling for the expected inaccuracies of these estimates may be obtained from straightforward statistical calculations. For example, if we estimate the correlation function of a Gaussian noise $n(t)$ (assumed mean zero and unit variance) as

$$
r_{n}(\tau)=\frac{1}{T} \int_{0}^{T} n(t) n(t+\tau) d t
$$

we find that the statistical mean $E\left(r_{n}(\tau)\right)=\rho_{n}(\tau)$, the true correlation function. The variance turns out to be (for $T \gg \tau_{0}$, the correlation time)

$$
\sigma_{r}^{2}=\sqrt{\frac{\pi}{2}} \frac{\tau_{0}}{T}\left(1+\rho_{n}^{2}(\tau)\right) .
$$

Thus except for a factor of the order of unity, the standard deviation of the correlation estimate depends inversely on the square root of the number of correlation times $T / \tau_{0}$ in the sample (the familiar TW of communication theory). A similar result is obtained for the distribution function by assuming that the $T / \tau_{0}$ correlation periods are equivalent to $n$ independent samples from the desired distribution (this is the usual correspondence between continuous and discrete probability calculations). Each sample can then be considered an independent Bernoulli trial with probability $p=P(A)$ that it has a value no greater than $A$. It is easily shown [Feller, 1957] that

$$
\sigma_{p}^{2}=\frac{p(1-p)}{n}=\frac{p(1-p) \tau_{0}}{T}
$$

with a maximum value $\tau_{0} / 4 T$. Both the correlation and distribution estimates are seen to have the same dependence on correlation and sample times. As an 
example, suppose that a $10-\mathrm{min}$ sample is taken of a function with a 15 -sec correlation time. Errors of the order of 0.2 sbould be expected in the normalized measured correlation, and distributions might be in error by almost 0.1. These figures should indicate the hazards of fitting distributions and estimating correlation times for the signals considered here.

The criterion chosen in determining correlation time is that time for which the correlation curve equals 0.5. Probably the simplest way to display the results is in a histogram, shown as figure 4. Here the records are separated only by whether the path was one-hop $E$, one-hop $F$ or multiple-hop $F$. The results for summer and winter conditions did not seem to differ appreciably and were accordingly taken together. From figure 4, it would appear that there is not a significant difference between the onehop $E$ - and $F$-layer results. Both give a most probable fading time of between 10 and 20 sec, with a comparable number less than 10 sec and a long tail corresponding to longer fading periods. Two of the records appeared to have correlation times of about 120 sec although, being comparable to the total length of the record, these figures should not be taken too seriously. Contrasted with this behavior, the multiple-hop paths nearly always gave fading times of a very few seconds. Presumably these would be the predominant signals in communication circuits over much greater distances and would account for the common impression of fairly rapid fading at HF.

Little of value can be stated on the shape of the correlation curves, most of which simply decreased from unity to values consistent with the expected departures from zero, with various types of irregularities occurring in many of the curves. In perhaps 10 or 15 cases, distinct indications appear of a decaying sinusoid in the correlation function, reflecting a regular, quasi-periodic fading pattern. (The subsequent peaks ordinarily did not reach 0.5 , but in all cases the first downward crossing of 0.5 was given as the correlation time. Thus, in some cases the signal may actually be correlated over even longer intervals than reported.)

It is natural at this point to inquire whether any consistent patterns appear in the data which might be compared with propagation theories. In particular, the data on several days consisted of records taken in as rapid succession as possible of frequencies all across the usable band. From calculations on plane waves in inhomogeneous media it would be expected, for instance, that for a given path, the signal-tonoise ratio ought to increase with frequency as the scattering decreases and that a trend with frequency in the fading time should be discernible which reflects the mechanism of scattering. It must, unfortunately, be reported that such was not the case. Both the form of the distribution function and the correlation time changed erratically as records (for example, of the $F$-layer return) were examined over a two-to-one band of frequencies during a time period of a couple of hours. This again must be largely ascribed to the nonstationarity of the ionosphere. Indeed on at least two occasions successive records
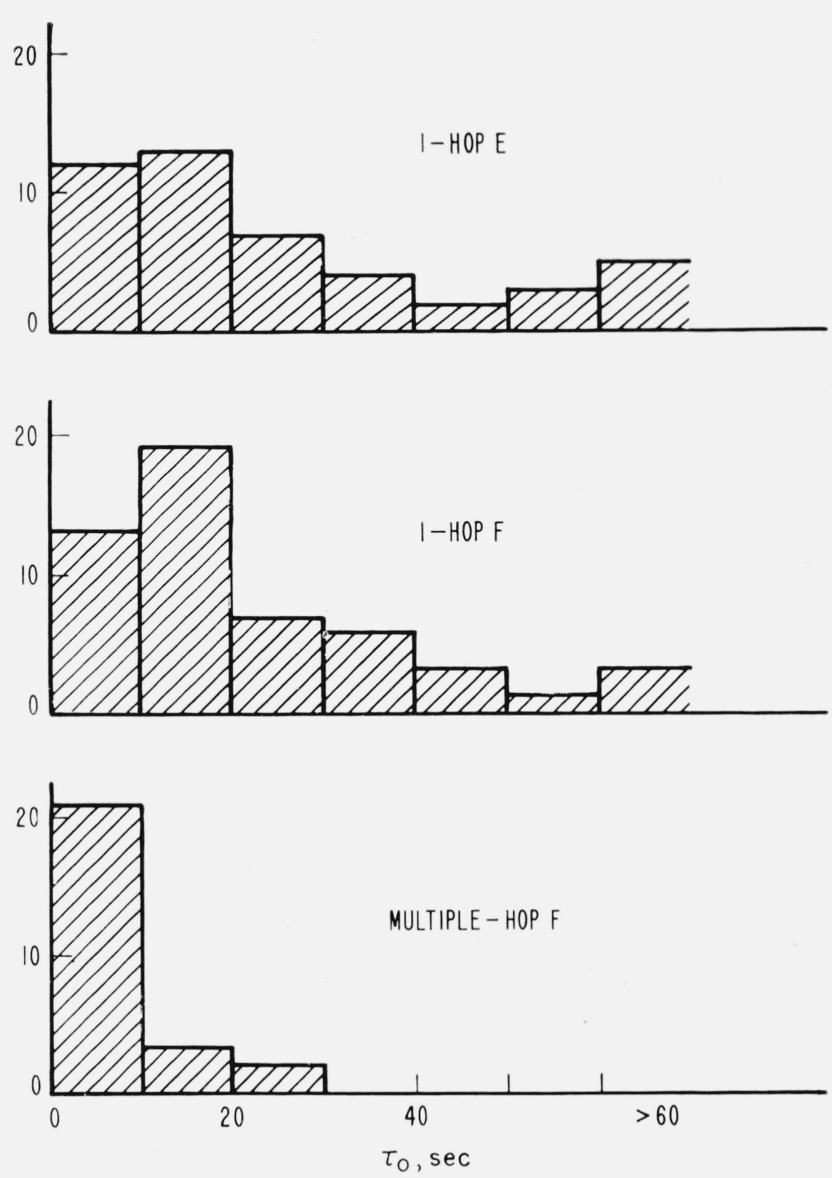

Figure 4. Histogram of correlation times.

were taken within $15 \mathrm{~min}$ of each other for the same path at the same frequency, with the result that the pairs of distributions were dissimilar and the fading times differed by a factor of about 2 . A condition which further complicates the task is the varying mixture of unresolved paths which are taken to be a single path. The effects computed for a pure path will of course be corrupted by the more complicated nature of the received signal. The results must therefore be taken as indicative of the conditions to be expected under normal propagation conditions, but not of the correct form to check theoretical calculations on stationary, single-path waves.

\section{Results-Space Correlation}

Of the results presented in the preceding section, 31 records were of the space correlation type mentioned in section 2. For these tests, six whip antennas were placed in a line perpendicular to the direction of propagation. The six signals were sampled simultaneously to study a given path, and recorded as the six channels in the data-processing system. (In the preceding section, these six records were presented as one, parameters being derived from a composite distribution and autocorrelation function. The individual curves, incidentally, show variations among them consistent with the earlier 
discussion on statistical fluctuations in estimated quantities.) The six antennas were spaced different integral numbers of hundreds of feet apart, thus affording 15 different antenna separations, the largest of which was $2,000 \mathrm{ft}$. The first aim was to check the space correlation distances and, in particular, the rule-of-thumb that this distance should be a few wavelengths. It was also hoped to obtain, if possible, more detail on the shape of the space correlation curve.

Except for two or three of the curves, the computed crosscorrelations were fairly consistent, viz, the points were scattered about a more or less well defined curve so that distances at which a given correlation occurred could be specified to within $100 \mathrm{ft}$ or so. Briefly stated, the principal result indicated by these crosscorrelation curves was that the correlation distances for the paths being studied was much greater than anticipated. In only seven of the 31 cases did the correlation curve reach below the value $\rho_{A}=0.5$ in less than the greatest distance measured, $2,000 \mathrm{ft}$. In the remainder of the cases, correlation distances could be determined only by extrapolation, and then in many records only approximately. For five of the curves, the correlation was greater than 0.9 at $2,000 \mathrm{ft}$, leading to an estimate of $10,000 \mathrm{ft}$ or more for the correlation distance.
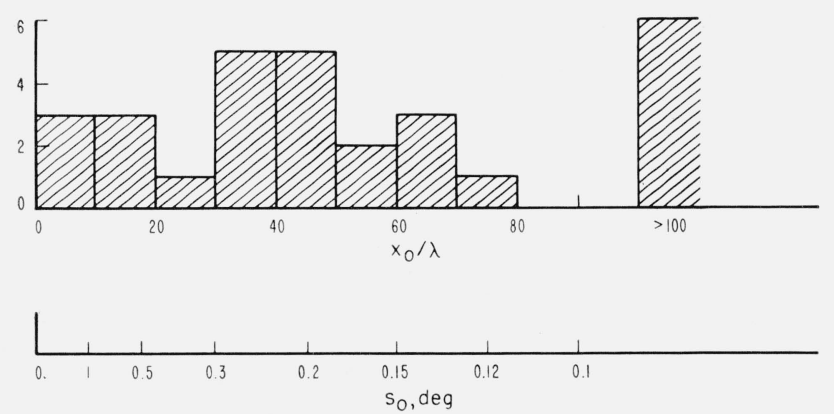

Figure 5. Histogram of correlation distances in wavelengths. The lower scale is a measure of the deviation angle $s_{0} \approx \frac{1}{9} \lambda / x_{0}$.

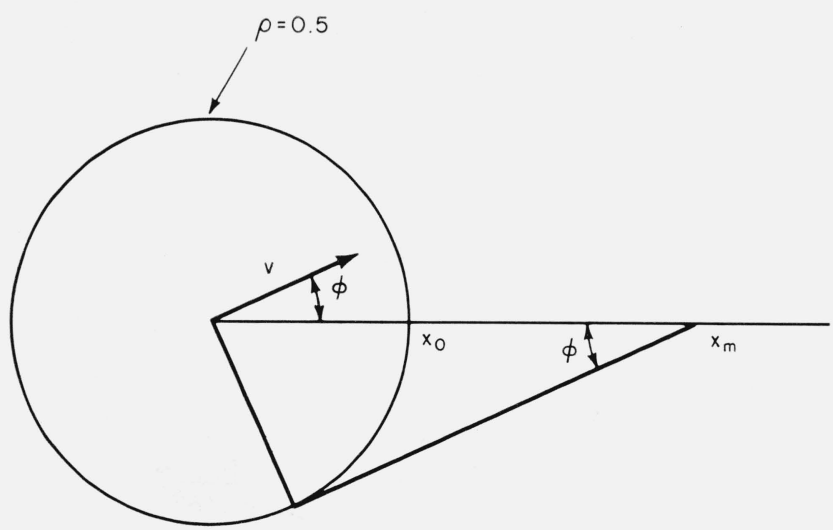

Figure 6. Geometry of translated field pattern.
Figure 5 is a histogram of the number of cases observed as a function of the correlation distance in wavelengths. It can be seen that the most common correlation distance is about 40 wavelengths. (A similar, though somewhat shorter, correlation distance is obtained from the more extensive mediumfrequency data by Brennan and Phillips [1957].) Presumably this marked difference from some earlier results [Van Wambeck and Ross, 1951] is due to the fact that isolated single-hop paths are considered, rather than mixed or multiple-hop signals. Of the three examples which yielded correlation distances of 10 wavelengths or less, one was a multiple-hop path (2-hop $F$, summer night) and the other two were $F_{1}$ (summer) paths. As these were the only multiplehop and $F_{1}$ paths included in the study, it would seem to indicate that these propagation paths do indeed exhibit shorter correlation distances.

Since the space correlation function is the Fourier transform of the angular spectrum of the received radiation [Booker, Ratcliffe, and Shinn, 1950], an indication of the mean angular deviation from the steady path may also be obtained from the preceding information. If, in particular, $\rho=\exp -\mathrm{x}^{2} / 2 \xi^{2}$ (where $\rho$ is the space correlation function of the total field, not just the amplitude), then the angular power spectrum is $P(s)=\exp -k^{2} s^{2} \xi^{2} / 2$, where $s=\sin \theta$. If the signal is a Gaussian noise, it can then be shown [Booker, Ratcliffe, and Shinn, 1950] that the amplitude correlation function $\rho_{A} \approx \rho^{2}$. It follows then that the half-power angle ( $s=\theta$ for small angles) $s_{0}=\frac{\ln 2}{2 \pi} \frac{\lambda}{x_{0}}$, where $x_{0}$ is determined, as in the results already given, by $\rho_{A}\left(x_{0}\right)=0.5$. This angle is then, apart from a constant, the reciprocal of the correlation length in wavelengths, and a corresponding scale may be found on the histogram in figure 5. (The constant of proportionality would vary somewhat with the shape of the correlation curve and with the addition of a specular component, but probably not by as much as a factor of 2.) The mean angular deviations obtained by this method - an average of perhaps $0.2^{\circ}$, with some summertime values of about $1^{\circ}$ —are in fair agreement with observed values of angle-of-arrival fluctuations by direction finders, e.g., by Bramley [1955].

Although this configuration of antennas in a single line was not designed to study ionospheric winds, clear indications of wind effects can be observed in that the peaks of the full crosscorrelation curves are found in just about half of the records to be displaced from the origin. A rather complete description of these effects is given by Briggs, Phillips, and Shinn [1950]. For the present purposes, the simplified picture of figure 6 for pure translation of field pattern (no internal motion of inhomogeneities) with isotropic ground correlation will suffice to illustrate the effects. Suppose the pattern is moving across the ground with a velocity $v$ at an angle $\phi$ with respect to the line of antennas. The autocorrelation function at a given site is then simply the space correlation function with the two abscissae converted through the velocity $v$. (As indicated earlier, the shapes of 
the correlation curves were not sufficiently well defined to test this statement directly.) In particular, if we denote the correlation in space and time by $\rho_{A}(x, \tau)$, we have already determined $x_{0}$ and $\tau_{0}$ by $\rho_{A}\left(x_{0}, 0\right)=\rho_{A}\left(0, \tau_{0}\right)=0.5$. It is then seen that $v=x_{0} / \tau_{0}$, since $\tau_{0}$, is the time it takes for the field at a distance $x_{0}$ to reach the origin and therefore give the same correlation in time as it gave in space. Now at each antenna separation, $x$, a maximum is reached in the crosscorrelation function at time $\tau_{m}(x)$, which indicates an apparent velocity $v^{\prime}=x / \tau_{m}(x)$. Examination of figure 6 (where, for example, $x_{m}$ is defined by $\left.\rho_{A}\left(x_{m}, \tau_{m}\left(x_{m}\right)\right)=0.5\right)$ shows that $v^{\prime}=v / \cos \phi$, which may be used to estimate $\phi$. Finally by finding the point $x_{m}$, another estimate of $\phi$ may be obtained from $x_{0}=x_{m} \sin \phi$.

An example of this procedure is given in figure 7 $(5-12-60,1148,21.35 \mathrm{Mc} / \mathrm{s}, E$.) The space correlation curve $\rho_{A}(x, 0)$, shown in figure $7 \mathrm{a}$, indicates $x_{0}=2,060 \mathrm{ft}$. (One channel was not operating that day, so only 10 rather than 15 points are available.) The composite autocorrelation function was used to find $\tau_{0}=5.8$ seconds, giving $v=355 \mathrm{ft} / \mathrm{sec}$. This would be twice the ionospheric speed which, converting units, is $54 \mathrm{~m} / \mathrm{sec}$. Most of the velocities came out some tens of $\mathrm{m} / \mathrm{sec}$, which are commonly measured ionospheric wind velocities.) Figure $7 \mathrm{~b}$ shows the delays $\tau_{m}$ at which the maxima appeared in the various crosscorrelation functions, indicating a velocity $v^{\prime}=425 \mathrm{ft} / \mathrm{sec}$ and hence an angle $\phi=33^{\circ}$. Finally, figure $7 \mathrm{c}$ is a plot of $\rho_{A}\left(x, \tau_{m}(x)\right)$. This curve may be extrapolated to where it crosses $\rho_{A}=0.5$ at $x_{m}=3,700 \mathrm{ft}$, giving the value $\phi=34^{\circ}$. This remarkable agreement is of course fortuitous and would not be expected either from the accuracy of the individual measurements or due to the fact that the correlation pattern is undoubtedly not isotropic for oblique paths. Other examples gave discrepancies between the two estimates of 10 to $15^{\circ}$, which must be considered more normal. It may thus be concluded that for a good fraction (of the order of $1 / 2$ ) of the time, even at oblique incidence the fading is due in large measure to the translation of the field pattern along the ground as a result of ionospheric wind drift.

\section{Conclusions}

Nearly 150 samples of HF pulse amplitude data, averaging about $10 \mathrm{~min}$ in length, were processed in an attempt to derive statistical parameters which might be used to describe or classify these signals. One important characteristic, the first order distribution of the amplitude, was obtained for each sample. In just about half of the cases, the distribution could be identified with a member of the family of distributions of a sine wave in gaussian noise, with most corresponding to pure noise (the Rayleigh distribution). A small number were identified with the amplitude of two sine waves beating in random phase. The other half of the distributions were of a different character, and seem to be best explained as being examples of nonstationary signals. Correlation times

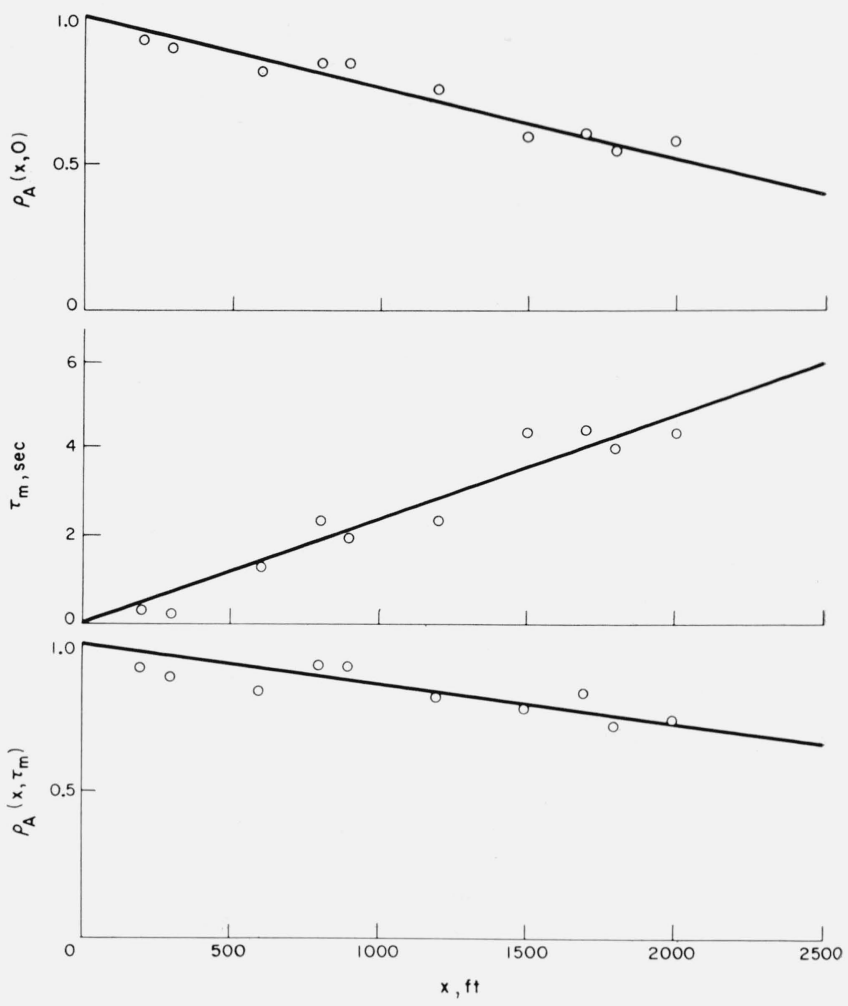

Figure 7. Example of ionospheric wind calculation.

(a) $\rho_{A}(x, 0)$, the amplitude space correlation function, (b) $\tau_{m}(x)$, the time displacement for maximum correlation, (c) $\rho_{A}\left(x, \tau_{m}\right)$, the correlation at the displaced times.

were also obtained for each record. Representative correlation (or fading) times were 20 sec for one-hop paths and a very few seconds for multiple-hop paths. The implication of these two results is that times over which the ionosphere can be considered to be a stationary medium may be only a few minutes in duration, and since the correlation time can be an appreciable fraction of a minute, it may often be possible to give only a rough statistical description of the signal at a given time.

Space correlation curves were obtained for 31 of the samples. The correlation distances thus obtained were of the order of 40 wavelengths for most of the single-hop paths studied and around 10 wavelengths only for a multiple-hop path and the $F_{1}$-paths. These figures correspond to angular fluctuations in the arriving signal of the order of 0.2 and $1^{\circ}$, respectively. Finally, many of these samples showed displaced maxima in the crosscorrelation curves, corresponding to ionospheric drifts of some tens of meters per second.

The authors are indebted to C. A. Wagner, R. E. Gay, and P. L. Fleck for their contributions to the data processing equipment, and to Mrs. C. K. McElwain and R. M. Cherry, who performed the digital data analysis. Thanks are also due W. B. Wrigley, W. Reagh, and G. Falcon for their help in operating the Atlanta terminal, and to T. S. Pitcher for several helpful discussions on statistical problems. 


\section{References}

Booker, H. G., J. A. Ratcliffe, and D. H. Shinn, Diffraction from an irregular screen with applications to ionospheric problems, Phil. Trans. Roy. Soc. A 242, 579 (1950).

Bramley, E. N., Some aspects of the rapid directional fluctuations of short radio waves reflected at the ionosphere, Proc. IEE B 102, 533 (1955).

Brennan, D. G., and M. L. Phillips, Phase and amplitude variability in medium-frequency ionospheric transmission, Lincoln Laboratory Tech. Rept. 93, 16 Sept. 1957.

Briggs, B. H., G. J. Phillips, and D. H. Shinn, The analysis of observations on spaced receivers of the fading of radio
signals,Proc. Phys. Soc. London, B63, 106 (1950).

Feller, W., An Introduction to Probability Theory and its Application, p. 214 (John Wiley \& Sons, Inc., New York, N.Y., 1957).

Nupen, W., Bibliography on ionospheric propagation of radio waves (1923-1960), NBS Tech. Note 84 (Oct. 1960).

Rice, S. O., Mathematical Anaylsis of Random Noise, Bell Syst. Tech. J. 24, 101 (1945).

Tatarski, V. I., Wave Propagation in a Turbulent Medium, p. 209 (McGraw-Hill Book Co., New York, N. Y., 1961). Van Wambeck, S. H., and A. H. Ross, Performance of diversity receiving systems, Proc. IRE 39, 256 (1951).

(Paper 66D6-232) 